\title{
Dynamique structurale des îlots de forêt claire à Anogeissus leiocarpa (DC.) Guill. et Perr. dans le Sud-Ouest du Burkina Faso
}

\author{
Zézouma SANON ${ }^{*}$, Mipro HIEN ${ }^{1}$, Jérôme T. YAMEOGO ${ }^{1}$, \\ Yvonne BACHMANN $^{2}$ et Irénée SOMDA ${ }^{1}$
}

${ }^{1}$ Laboratoire des Systèmes Naturels, des Agro-Systèmes et de l'Ingénierie de l'Environnement (Sy.N.A.I.E), Institut du Développement Rural (IDR), Université Polytechnique de Bobo-Dioulasso (UPB), Burkina Faso.

${ }^{2}$ Institute for Ecology, Evolution and Diversity, University Frankfurt am Main (GUF), Germany.

*Auteur correspondent, E-mail: zezoumasanon@yahoo.fr

\section{RESUME}

Les forêts claires résultent de la dégradation des forêts denses sèches. Elles sont soumises aux facteurs climatiques et anthropiques. Cette étude vise à analyser la diversité floristique et la dynamique des principales espèces dans la forêt classée de Koulbi. Les données ont été collectées dans 27 placeaux de $900 \mathrm{~m}^{2}$. Le diamètre $\left(\mathrm{Dbh}_{1,30 \mathrm{~m}}\right)>5 \mathrm{~cm}$ et la hauteur des individus ont été mesurés. La régénération a été comptée et l'état sanitaire apprécié. Les indices de Shannon et de Piélou, la valeur d'importance écologique de l'espèce (IVI), la densité et la surface terrière ont été calculés. La distribution de Weibull a été appliquée à la structure des principales espèces. L'indice de Shanonn $=3,01$ bits montre une diversité moyenne et l'indice de Pielou $=$ 0,49 traduit la dominance de quelques espèces. Les espèces ayant un IVI significatif sont Anogeissus leiocarpa (DC.) Guill. Et Perr. (116,19), Pterocarpus erinaceus Poir. (21,66), Lannea barteri Oliv. Engl. (13,40), Adansonia digitata L. (12,92) et Vitellaria paradoxa Gaertn. (11,80). La principale espèce, A. leiocarpa présente une bonne densité tant au niveau des adultes (316 pieds/ha) que des juvéniles (667 pieds/ha). Sa structure diamétrique en «J renversé» est synonyme d'une bonne dynamique. Cependant, elle est menacée par la mortalité $(9,27 \%)$ par endroit des sujets.

(C) 2015 International Formulae Group. All rights reserved.

Mots clés : Diversité floristique, régénération, mortalité, forêt classée de Koulbi.

\section{INTRODUCTION}

Les forêts claires sont le résultat d'une dégradation des forêts denses sèches et se maintiennent dans cet état du fait des feux de brousse et de l'existence d'une saison sèche suffisamment longue (Bellefontaine et al., 2000). Dans le Sud-Ouest du Burkina Faso, elles sont rencontrées sous forme de reliques sur de petites superficies (Fontes et Guinko,
1995). Ces îlots se font rares de nos jours hors des zones de conservation. Dans la forêt classée de Koulbi, elles sont dispersées en de petites taches dans des zones de dépressions et le long du Mouhoun et ses affluents. Selon Tankoano (2012), leur superficie a connu une réduction de 25,88\% entre 1986 et 2006 dans la forêt classée de Koulbi. Les causes seraient la pression anthropique et les changements 
climatiques en cours. En effet, la région du Sud-Ouest constitue une zone d'accueil des migrants agricoles en provenance du Centre et du Nord du pays (Balma et al., 2004). De même, pour Courtin et al. (2010), suite à la crise ivoirienne de 2002, près de trois cent soixante mille burkinabé sont rentrés. Une bonne partie serait restée dans le Sud du pays, notamment dans les communes frontalières comme Kpéré et Batié, où les conditions agroclimatiques sont plus favorables et proches de celles de la Côte d'Ivoire. Cela a contribué à mettre en mal l'état de protection de la forêt classée de Koulbi. Cette forêt abritait des villages et une population importante dont l'activité principale était l'agriculture itinérante sur brulis avant le déguerpissement de 2009.

Au titre du climat, nous notons que les variabilités climatiques en cours dans les zones arides et semi-arides ne sont pas sans conséquence sur la dynamique des écosystèmes forestiers en général et sur les reliques de forêts claires en particulier. En effet, Adjonou et al. (2009) rapportent dans des conditions climatiques similaires aux nôtres une forte mortalité des individus de $A$. leiocarpa dans les forêts claires du parc National Oti-kéran au Nord du Togo.

En dépit de tous ces facteurs préjudiciables à la bonne dynamique des écosystèmes de la forêt classée de Koulbi, peu d'investigations scientifiques ont porté sur cette forêt classée. Cette étude sur les vestiges des forêts claires à A. leiocarpa se veut être une contribution à une meilleure connaissance de la diversité floristique et de la dynamique des forêts claires de la forêt classée de Koulbi.

\section{MATERIEL ET METHODES Milieu d'étude}

La forêt classée de Koulbi est située dans l'extrême sud du Burkina Faso entre $2^{\circ} 58^{\prime}-2^{\circ} 42^{\prime}$ de longitude Ouest et $9^{\circ} 29^{\prime}$ $9^{\circ} 45^{\prime}$ de latitude Nord. Classée par l'Arrêté 387/FOR du 4 août 1955, elle s'étend sur 40 000 ha. Elle est limitée à l'Ouest par la République de Côte d'Ivoire et à l'Est par le fleuve Mouhoun (Figure 1). Selon le découpage phytogéographique de Fontes et Guinko (1995), le site d'étude appartient au secteur sud-soudanien. La moyenne pluviométrique de 1971 à 2010 est de 1057 $\mathrm{m} / \mathrm{an}$. La tendance de la pluviométrie des 40 dernières années reste stable (Figure 2). Pendant la même période la température moyenne est de $27,9^{\circ} \mathrm{C}$. Contrairement à la pluviométrie, on note une augmentation de la température (Figure 3). Du fait de sa situation, la végétation est dominée par les savanes auxquelles s'associent des reliques de forêts claires de faibles superficies (Fontes et Guinko 1995). La forêt classée est à cheval entre la commune de Batié et celle de Kpéré dont les taux de croissance démographique entre 1996 et 2006 sont respectivement de $3,8 \%$ et $6,7 \%$ (ISND, 2009).

\section{Collecte de données}

\section{Données de végétation et du milieu physique}

Les données ont été collectées dans 27 placeaux circulaires de $900 \mathrm{~m}^{2}$. Le diamètre de tous les individus dont le diamètre à hauteur de poitrine $(\mathrm{dbh}) \geq 5 \mathrm{~cm}$ a été mesuré avec un ruban et leur hauteur estimée. Pour rendre compte de la dynamique de la régénération, 5 placettes de $25 \mathrm{~m}^{2}$ ont été installées dans le placeau principal (Figure 4). La régénération a été comptée par espèce et classée suivant des classes de hauteurs $(\mathrm{m})$ :] $0-0,5] ; 0,5-1] ;$; 1-1,5] et] 1,5-2]. Le taux de recouvrement global des ligneux a été également noté. La flore de Arbonnier (2009) et celle de Mugnier (2008) ont servi à l'identification des espèces.

\section{Etat sanitaire des forêts claires}

L'évaluation de l'état sanitaire a consisté à enregistrer les individus morts de chaque espèce lors de l'inventaire. Aussi, des observations et des prélèvements d'échantillons d'insectes ont été faits sur 5 sujets visuellement sains, sur 5 sujets moribonds et sur 5 sujets morts de $A$. leiocarpa car cette espèce présentait des signes d'attaque et de mortalité. Les diamètres et les hauteurs ont été mesurés. Les insectes et 
autres indices observés sur le tronc ont été notés et le nombre de perforations sur le tronc a été compté dans un carré de $10 \mathrm{~cm}$ de côté.

\section{Analyse statistique des données}

Pour rendre compte de la diversité floristique, l'indice de Shannon ( $\left.\mathrm{H}^{\prime}\right)$, l'indice d'équitabilité de Pielou (Eq) et la valeur d'importance écologique de l'espèce (IVI) ont été calculés :

L'indice de Shannon (H') est faible lorsqu'il est compris entre $0 \leq \mathrm{H}^{\prime} \leq 2,5$, cela se traduit dans la communauté par la dominance d'une seule espèce ou d'un petit nombre d'espèces. Lorsque $2,6 \leq \mathrm{H}^{\prime} \leq 3,9$, il peut être supposé moyen. Et lorsque $4 \leq \mathrm{H}^{\prime}<$ 6, il est considéré comme élevé, par conséquent, les espèces tendent vers l'équiprobabilité.

$$
H^{\prime}=-\sum_{i=1}^{s} p_{i} \log _{2} p_{i}
$$

Avec $\mathrm{P}_{\mathrm{i}}=\mathrm{n}_{\mathrm{i}} / \mathrm{n}$ où $\mathrm{n}_{\mathrm{i}}=$ nombre d'individus d'une espèce $\mathrm{i}$ et $\mathrm{n}=$ nombre total d'individus dans le placeau, $S=$ richesse spécifique et $\log _{2}=\log$ arithme à base 2 .

L'indice de Pielou mesure la régularité ou l'équitabilité de l'abondance des espèces. Si $0 \leq \mathrm{Eq} \leq 0,6$ alors $\mathrm{Eq}$ est faible, par conséquent il existe le phénomène de dominance dans la communauté. Si $0,7 \leq \mathrm{Eq}<$ 0,8 alors Eq est moyenne. Si $0,8 \leq \mathrm{Eq} \leq 1$, alors Eq est élevée, par conséquent on a une absence de dominance dans la communauté.

$$
\mathrm{Eq}=\frac{\mathrm{H}^{\prime}}{\log _{2} \mathrm{~S}}
$$

La valeur d'importance écologique d'une espèce (IVI) est la somme de sa dominance relative (Domr, de sa densité relative $(\mathrm{Dr})$ et de sa fréquence relative (Fr) $(\mathrm{IVI}=\mathrm{Domr}+\mathrm{Dr}+\mathrm{Fr})$. IVI est un indice quantitatif permettant d'identifier les espèces écologiquement importantes dans une communauté végétale (Adomou, et al., 2009 ; Dossou et al., 2012). Elle varie de 0 (absence de dominance) à 300 (mono-dominance). Une espèce a une importance écologique lorsque son IVI> 10\% (Reitsma, 1988).

$$
\begin{aligned}
& \text { Domr }=\frac{\text { Surface terrière espèce }}{\text { Surface terrière toutes les espèces }} \times 100 \\
& \operatorname{Dr}=\frac{\text { Nombre individus espèce }}{\text { Nombre total individus }} \times 100 \\
& \mathrm{Fr}=\frac{\text { Fréquence espèce }}{\text { Somme des fréquences }} \times 100
\end{aligned}
$$

\section{Analyse de la structure des espèces}

Le diamètre quadratique (d) a été utilisé pour les individus fourchus avant 1,30 $\mathrm{m}$ du sol. Il se veut plus précis que la moyenne arithmétique dans la détermination du diamètre moyen des arbres multicaules (Gouwakinnou et al., 2009).

$$
d=\sqrt{\sum_{i=1}^{n} d s i^{2}}
$$

Avec dsi : diamètre fourche $i$ en $\mathrm{cm} ; \mathrm{n}$ : nombre de brins constitutifs de l'arbre.

La densité des arbres (N), a été calculée suivant la formule :

$$
\mathrm{N}=\frac{\mathrm{n}}{\mathrm{S}}
$$

n: nombre total d'individus ; $\mathrm{S}=$ unité d'échantillonnage en ha.

La surface terrière $(\mathrm{G})\left(\mathrm{m}^{2} / \mathrm{ha}\right) \mathrm{du}$ peuplement est la somme des surfaces terrières de tous les arbres qui constituent le peuplement. Elle est obtenue par la formule :

$$
\mathrm{G}=\frac{\pi}{4 \mathrm{~s}} \sum_{\mathrm{i}=1}^{\mathrm{n}} \mathrm{d}_{\mathrm{i}}^{2}
$$

di : dbh de l'individu « $\mathrm{i} » ; \mathrm{n}$ : nombre d'individus 
Le diamètre moyen d'un individu (cm), a été calculé à partir de la formule proposé par Van Laar et Akça (2007)

$D=\sqrt{\frac{1}{n}} \sum_{i=1}^{n} d i^{2}$

La hauteur moyenne d'un individu a été obtenue en utilisant la hauteur moyenne de Lorey (Philip, 2002).

$$
\mathrm{H}=\frac{\sum_{\mathrm{i}=1}^{\mathrm{n}} \mathrm{g}_{\mathrm{i}} \mathrm{h}_{\mathrm{i}}}{\sum_{\mathrm{i}=1}^{\mathrm{n}} \mathrm{g}_{\mathrm{i}}}
$$

gi : surface terrière $\left(\mathrm{m}^{2} / \mathrm{ha}\right)$ de l'individu « $\mathrm{i}$ »; hi : hauteur $(\mathrm{m})$ de l'individu « $\mathrm{i}$ »

Des auteurs tels que Assogbadjo et al. (2009) ; Glélè Kakaï et al. (2011) ont eu recours à ces formules pour calculer le diamètre moyen et la hauteur moyenne car elles ont l'avantage d'être plus stables que la moyenne simple.

Le logiciel Minitab 16, a été utilisé pour appliquer la distribution de Weibull à trois paramètres sur les données de diamètres des espèces caractéristiques. La distribution de Weibull à 3 paramètres $(\mathrm{a}, \mathrm{b}$ et $c$ ) se caractérise par une grande souplesse d'emploi et une grande variabilité de forme (Rondeux, 1999). Pour chaque espèce, les diamètres des arbres ont été utilisés pour l'estimation des paramètres $(\mathrm{a}, \mathrm{b}$, et c) grâce à un algorithme basé sur la méthode du maximum de vraisemblance (Zarnock et Dell, 1985). Le paramètre « $\mathrm{a} »$ détermine la position, le paramètre $« b »$ détermine l'échelle et le paramètre 《 $\mathrm{c} »$ détermine la forme liée à la structure observée. Suivant la valeur de «c», nous avons les formes de distribution de Weibull suivantes (Ryniker et al., 2006) :

- c < 1 : distribution en « $\mathrm{J}$ renversé », caractéristique des peuplements multispécifiques ou inéquiennes ;

- $c=1$ : distribution exponentiellement décroissante, caractéristique des populations en extinction ;

- $1<\mathrm{c}<3,6$ : distribution asymétrique positive ou asymétrique droite, caractéristique des peuplements mono-spécifiques avec prédominances de faible diamètre ;

- $\mathrm{c}=3,6$ : distribution symétrique ; structure normale, caractéristique des peuplements équiennes ou monospécifiques de même cohorte ;

- c > 3,6: distribution asymétrique négative ou asymétrique gauche, caractéristique des peuplements monospécifiques à prédominance d'individus de gros diamètres.

$$
g_{i}=\frac{\pi}{4} d^{2}
$$

\section{RESULTATS \\ Composition et diversité floristique des espèces ligneuses}

Sur l'ensemble des relevés dans les forêts claires, nous avons recensé 67 espèces ligneuses reparties en 54 genres et 28 familles. Les familles les plus représentées sont les Mimosaceae (12\%), les Caesalpiniaceae $(10,4 \%)$, les Combretaceae $(10,4 \%)$, les Fabaceae (6\%), et les Rubiaceae (6\%). Les autres familles contribuent chacune pour moins de $5 \%$.

Les espèces ayant une importance écologie dans cette formation sont $A$. leiocarpa, P. erinaeus, L. barteri, A. digitata, et $V$. paradoxa (Tableau 1). Au nombre de 5 espèces, elles occupent plus de $50 \%$ de IVI total. L'espèce A. leiocarpa $(\mathrm{IVI}=116,19)$ est l'espèce la mieux représentée.

L'indice de Shannon $\left(\mathrm{H}^{\prime}=3,01\right.$ bits) est moyen alors que l'équitabilité de Pielou $(\mathrm{Eq}=0,49)$ est faible. Ces valeurs montrent la dominance de ces îlots par une ou quelques espèces.

\section{Densité et paramètres dendrométriques des principales espèces}

La densité moyenne (N) de toutes les espèces est de 538 pieds/ha. La contribution des espèces caractéristiques à cette densité est de 68,77\% ; A. leiocarpa avec une densité moyenne de 316 pieds/ha est le principal contributeur (Tableau 2). Cette même 
tendance est observée au niveau de la surface terrière où les quatre principales espèces contribuent pour plus de $60 \%$ de la surface terrière totale.

Les forêts claires présentent une forte régénération $(\mathrm{Nr}=3531$ plants/ha $) . A$. leiocarpa (667 pieds/ha) et $V$. paradoxa (551 pieds/ha) sont les espèces caractéristiques qui présentent une bonne régénération. Cependant, la contribution des espèces caractéristiques reste faible $(37,43 \%)$ (Tableau 2). Cela signale une forte contribution de certaines espèces à la génération, mais qui sont absentes dans la strate supérieure.

Les valeurs moyennes calculées des paramètres dendrométriques des principales espèces montrent que $L$. barteri et $P$. erinaceus ont un diamètre moyen supérieur à $30 \mathrm{~cm}$. Les deux autres espèces (A. leiocarpa et $V$. paradoxa) enregistrent un diamètre moyen inférieur à $20 \mathrm{~cm}$. Par contre, la strate verticale est dominée par $P$. erinaceus et $A$. leiocarpa respectivement 20,35 m et 19,14 m, suivis de L. barteri $(14,54 \mathrm{~m})$. V. paradoxa $(9,17 \mathrm{~m})$ occupe la strate inférieure (Tableau 2).

Les courbes de régression traduisant la relation de la croissance en hauteur en fonction de la croissance en diamètre font ressortir que le lien entre le diamètre d'un individu et sa hauteur est plus fort chez $A$. leiocarpa $\left(\mathrm{R}^{2}=0,68\right)$ et plus faible chez $V$. paradoxa $\left(\mathrm{R}^{2}=0,50\right)$ (Figure 5).

\section{Structure en diamètres des principales espèces}

La répartition des sujets par classes de diamètres de A. leiocarpa (Figure 6) présente une allure en « J renversé ». Cette distribution s'ajuste à la fonction de densité de Weibull avec le paramètre de forme $\mathrm{c}<1$, caractéristique des peuplements multispécifiques ou inéquiennes. Le peuplement de A. leiocarpa des forêts claires montre une forte prédominance des individus jeunes. Par contre, la distribution des individus par classes de diamètre de $P$. erinaceus, $L$. barteri et $V$. paradoxa est plus ou moins en cloche avec le paramètre de Weibull $1<\mathrm{c}<3,6$, correspondant à une distribution asymétrique positive ou asymétrique droite, caractéristique des peuplements mono-spécifiques (Figure 6). Chez $P$. erinaceus et $L$. barteri on note une faible présence des individus de petit diamètre.

\section{Structure de la régénération des principales espèces \\ Les espèces A. leiocarpa (667 pieds/ha)} et $V$. paradoxa (551 pieds/ha) présentent une bonne régénération comparativement à $P$. erinaeus ( 80 pieds $/ \mathrm{ha}$ ) et $L$. barteri (24 pieds/ha) (Tableau 2). Cependant, pour toutes ces espèces, l'essentiel de cette régénération se concentre dans la classe ] 0-0,5 m] (Figure 7). Le taux de survie est très faible, occasionnant une forte chute de la densité de plants entre la strate] $0-0,5 \mathrm{~m}$ ] et la suivante. Pour P. erinaceus et $L$. barteri, on constate une absence d'individus dans les classes supérieures (Figure 7). Les conditions climatiques et/ou la pression anthropique pourraient être à l'origine de cette mauvaise dynamique.

\section{Etat sanitaire des peuplements de forêts claires}

Les forêts claires à A. leiocarpa sont sous forte pression anthropique, surtout celles situées à la périphérie. Les îlots parcourus présentent $18,5 \%$ des signes d'anthropisation (Figure 8 ). En plus, la principale espèce ( $A$. leiocarpa) connait une mortalité localisée de l'ordre de 9,27\%. Des observations sur le tronc des sujets ont permis de noter un nombre important de perforations (2000 perforations $/ \mathrm{m}^{2}$ ) sur les sujets morts, des perforations sur les sujets moribonds avec présence d'insectes (Figure 9) et une absence d'insectes et de perforations sur les sujets sains. Le caractère localisé de la mortalité laisse croire à une attaque parasitaire ou virale. 


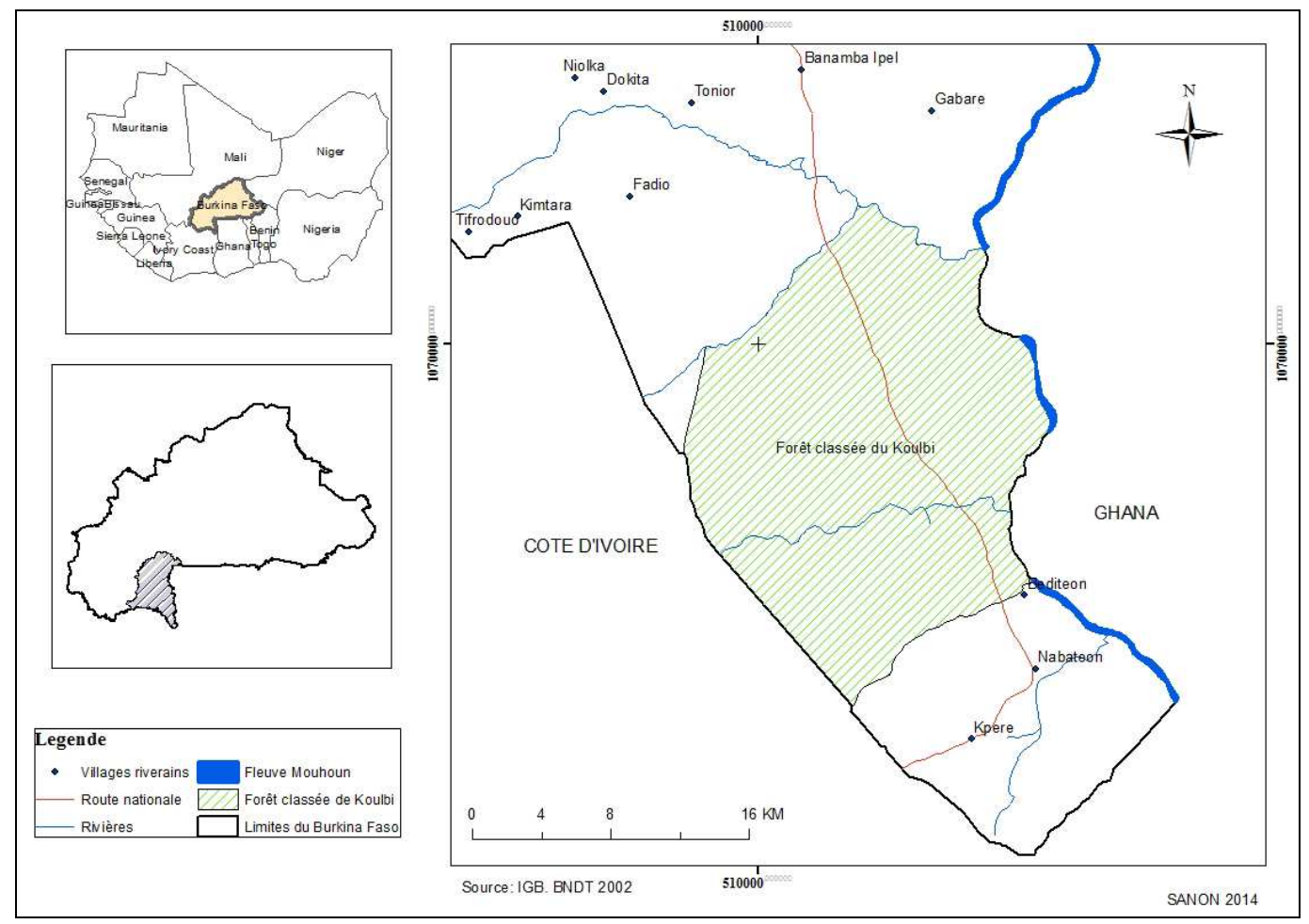

Figure 1: Localisation du site d'étude (Forêt classée de Koulbi).

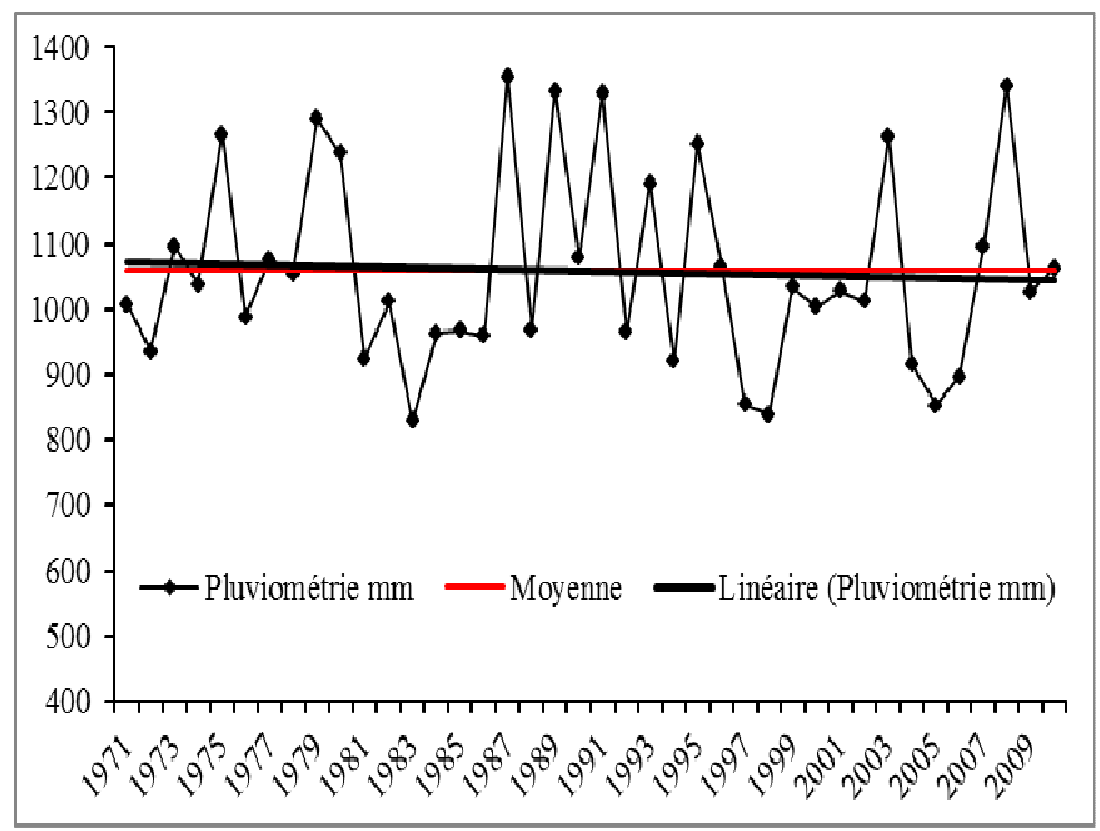

Figure 2: Variation pluviométrique de 1970 à 2010. 


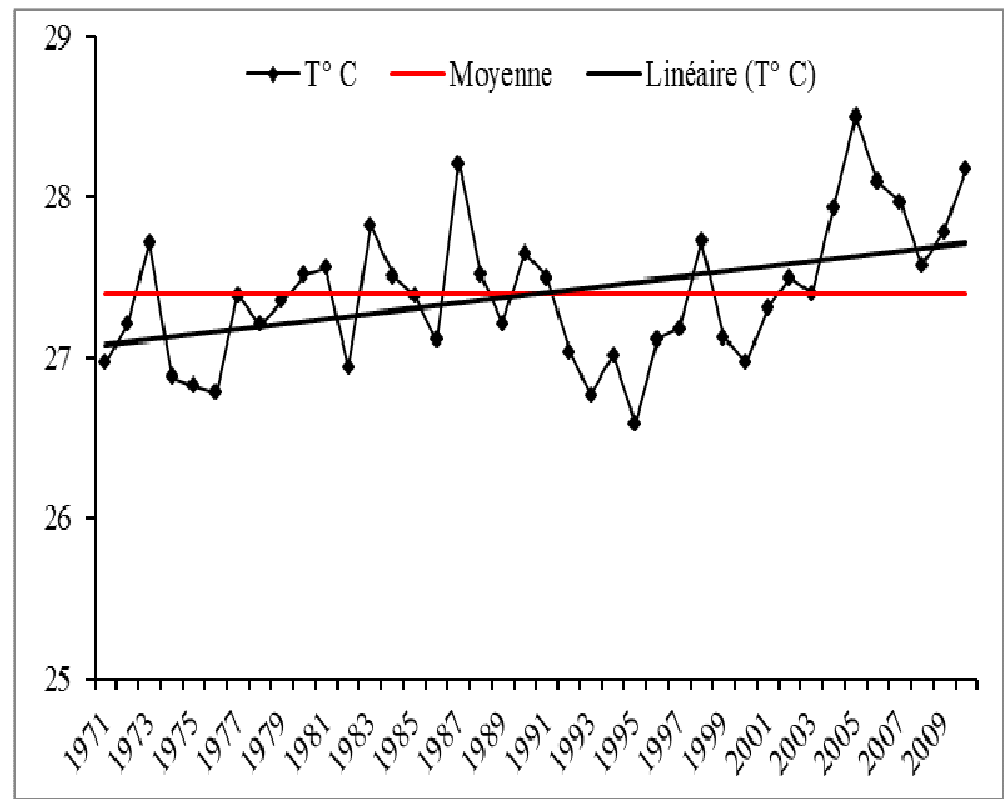

Figure 3: Variation de la température de 1970 à 2010.

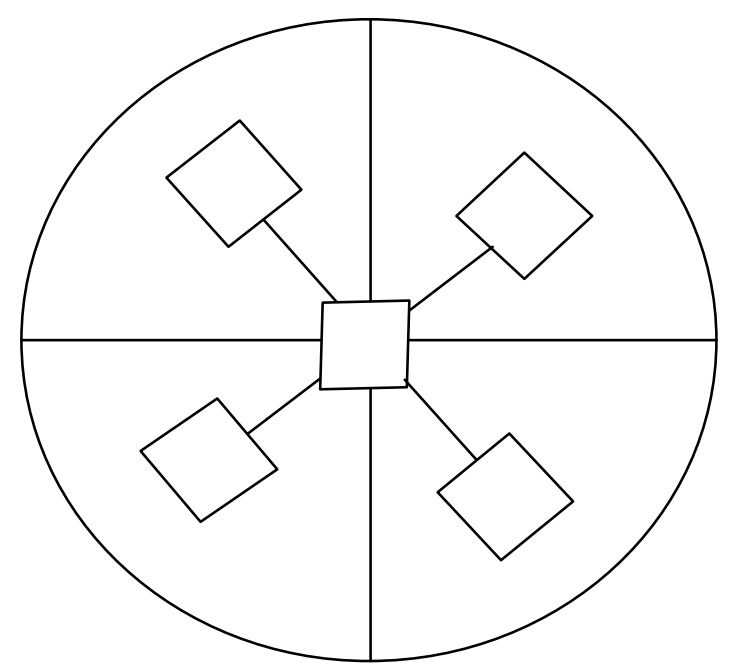

Figure 4: Représentation du placeau et des placettes d'inventaire. 

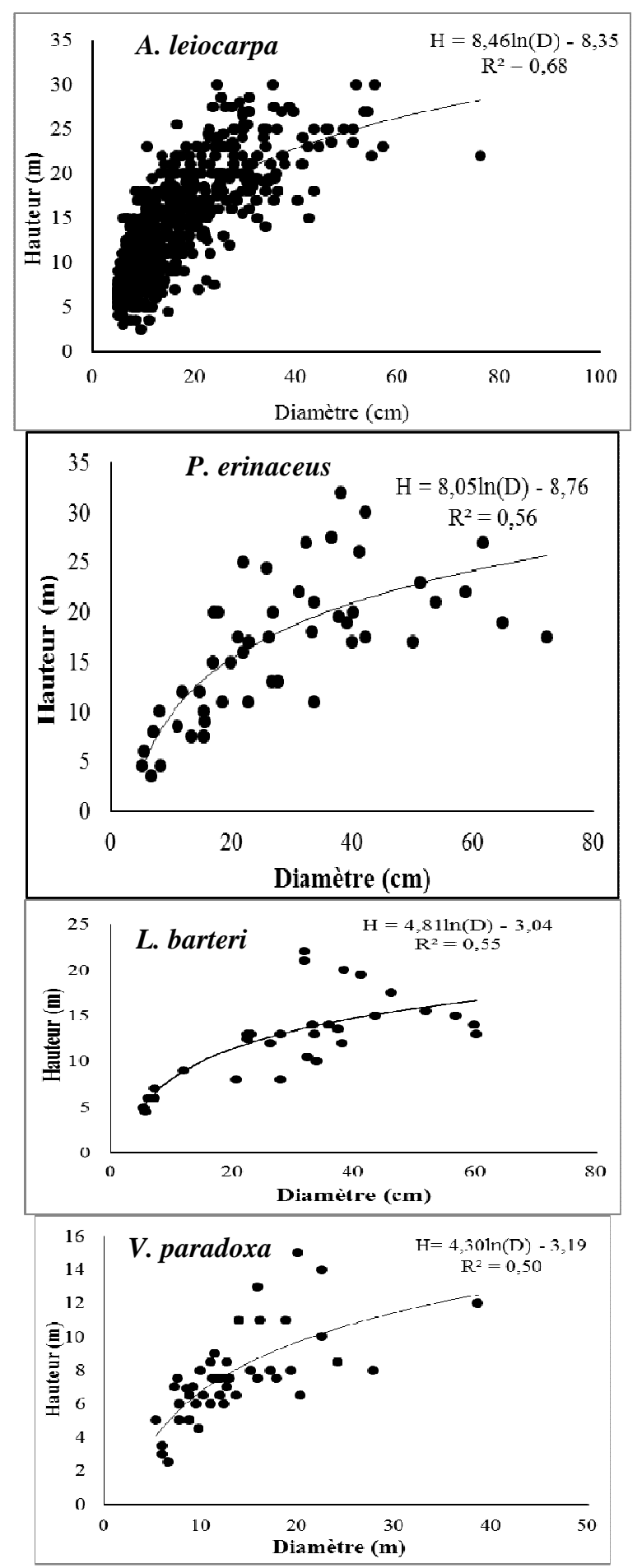

Figure 5: Courbe de régression traduisant la relation de la croissance en hauteur en fonction du diamètre. 


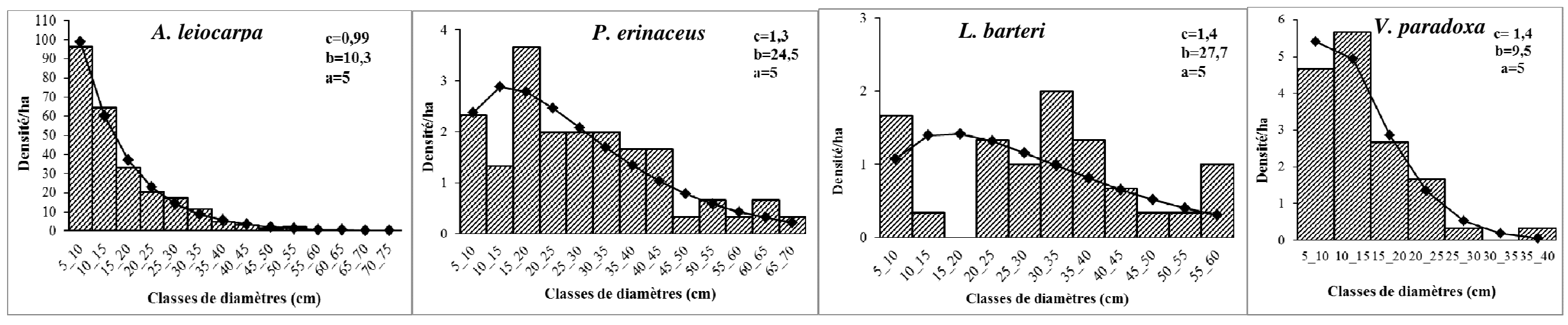

Figure 6: Dynamique de la structure des principales espèces ligneuses des îlots de forêts claires.
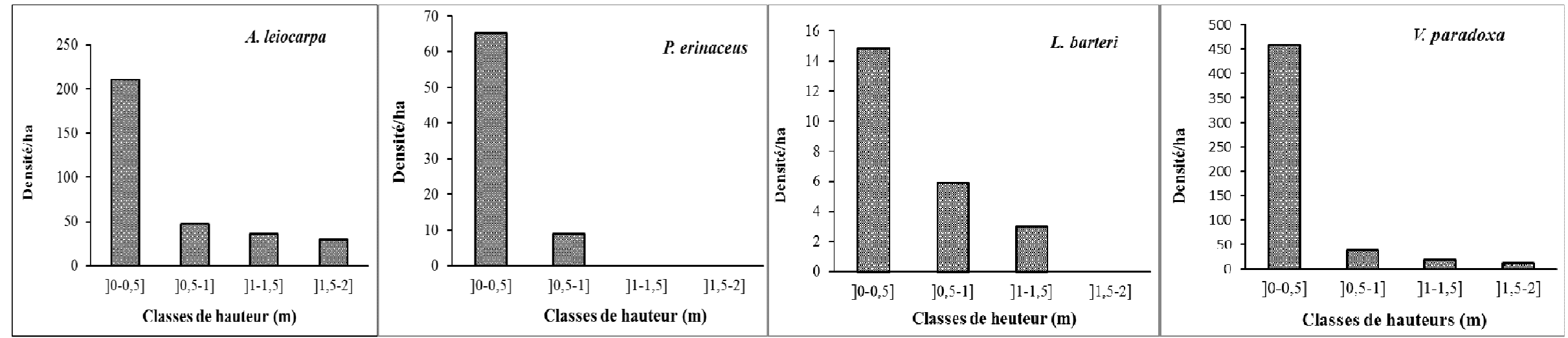

Figure 7: Dynamique de la structure de la régénération des principales espèces ligneuses des îlots de forêt claire. 


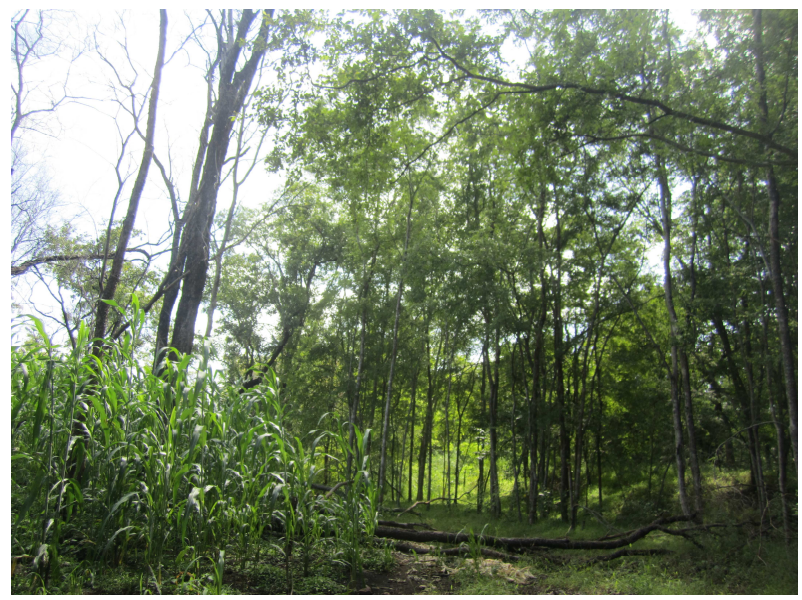

Figure 8: Pression anthropique sur les îlots de forêt claire à A. leiocarpa.

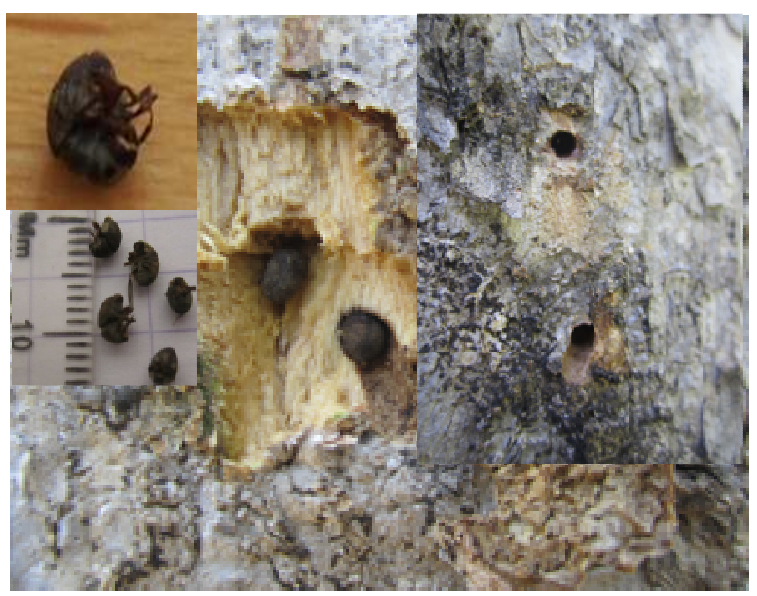

Figure 9: Attaques des insectes sur le tronc de $A$. leiocarpa.

Tableau 1: Valeur d'importance écologique de l'espèce (IVI).

\begin{tabular}{lcccc}
\hline Espèces & Domr & Dr & Fr & IVI \\
\hline Anogeissus leiocarpa (DC.) Guill. \& Perr. & 45,94 & 58,72 & 11,54 & 116,19 \\
Pterocarpus erinaceus Poir. & 10,46 & 4,36 & 6,84 & 21,66 \\
Lannea barteri Oliv. Engl. & 6,41 & 2,29 & 4,70 & 13,40 \\
Adansonia digitata L. & 12,42 & 0,08 & 0,43 & 12,92 \\
Vitellaria paradoxa Gaertn & 1,87 & 3,52 & 6,41 & 11,80 \\
Autres espèces & 22,90 & 31,04 & 70,09 & 124,03 \\
Total & $\mathbf{1 0 0}$ & $\mathbf{1 0 0}$ & $\mathbf{1 0 0}$ & $\mathbf{3 0 0}$ \\
\hline
\end{tabular}

Domr $=$ Dominance relative $;$ Dr $=$ Densité relative; Fr = Fréquence relative

Tableau 2: Caractéristiques dendrométriques des principales espèces.

\begin{tabular}{lccccc}
\hline Espèces & $\begin{array}{c}\mathbf{N} \\
\text { (pieds/ha) }\end{array}$ & $\mathbf{N r}$ (pieds/ha) & $\mathbf{G}\left(\mathbf{m}^{\mathbf{2}} / \mathbf{h a}\right)$ & $\mathbf{D}(\mathbf{c m})$ & $\mathbf{H}(\mathbf{m})$ \\
\hline Anogeissus leiocarpa & 316 & 667 & 8,26 & 18,24 & 19,14 \\
Pterocarpus erinaceus & 23 & 80 & 1,88 & 31,96 & 20,35 \\
Lannea barteri & 12 & 24 & 1,15 & 34,48 & 14,54 \\
Vitellaria paradoxa & 19 & 551 & 0,34 & 14,88 & 9,17 \\
Part des principales espèces & $\mathbf{6 8 , 7 7}$ & $\mathbf{3 7 , 4 3}$ & $\mathbf{6 4 , 6 8}$ & --- & --- \\
$(\%)$ & & & & \\
\hline Toutes espèces & $\mathbf{5 3 8}$ & $\mathbf{3 5 3 1}$ & $\mathbf{1 7 , 9 8}$ & $\mathbf{2 0 , 6 3}$ & $\mathbf{1 9 , 2 3}$ \\
\hline N= densité adulte ; Nr= densité régénération $; \mathrm{G}=$ surface terrière $; \mathrm{D}=$ diamètre moyen $; \mathrm{H}=$ hauteur moyenne.
\end{tabular}




\section{DISCUSSION}

Diversité et dynamique de la structure des forêts claires

Les résultats de cette étude fournissent des informations importantes sur la connaissance des forêts claires de la forêt classée de Koulbi. S'intéressant uniquement aux espèces ligneuses, la richesse spécifique est de 67 espèces. Les Combretaceae, les Mimosaceae et les Ceasalpiniaceae occupent une place importante parmi les familles qui colonisent ce type d'écosystème. Si nos résultats sont comparables à ceux de Adjonou et al. (2009) dans le Nord du Togo par la famille des Combretaceae (16), ils différent avec l'importance des Rubiaceae (23) notée dans son étude. Par contre, les résultats de cette étude corroborent ceux de Nacoulma (2012) dans le parc W. Les îlots de forêts claires à A. leiocarpa sont de petites superficies et subissent de ce fait une intrusion des espèces et des familles qui ne seraient pas caractéristiques de ces formations. En dépit de cela, il est à noter que les forêts claires présentent une faible diversité se traduisant par la dominance de l'espèce $A$. leiocarpa. La valeur d'importance écologique, la densité et la surface terrière de l'espèce témoignent de son importance dans la formation.

De façon globale, les valeurs de la densité moyenne (778 arbres/ha) et de la surface terrière $\left(20,29 \mathrm{~m}^{2} / \mathrm{ha}\right)$ de Adjonou et al. (2009) dans le Nord du Togo et celles de Sopkon et al. (2006), densité moyenne de 279 arbres/ha et surface terrière de $14,1 \mathrm{~m}^{2} / \mathrm{ha}$ diffèrent des résultats obtenus dans cette étude. Par contre, ces résultats s'approchent de celles de Paré (2008) obtenues dans une région voisine (Centre-Ouest du Burkina) de notre zone d'étude. Les espèces caractéristiques occupent $68,77 \%$ de la densité globale de la formation. A. leiocarpa, $L$. barteri, $P$. erinaceus et $V$. paradoxa sont les principales espèces qui contribuent fortement à la densité et à la surface terrière de la formation. Les forêts claires décrites par Adjonou et al. (2009); Sopkon et al. (2006) dans le domaine soudanien sont dominées également par une ou un nombre restreint d'espèces.

La dynamique structurale des espèces écologiquement importantes n'est pas la même pour toutes les espèces. A. leiocarpa présente une distribution par classe de diamètres en « $\mathrm{J}$ renversé », alors que les autres espèces ( $P$. erinaceus, $L$. barteri, et $V$. paradoxa) ont une distribution plus ou moins en cloche. La dynamique de A. leiocarpa est bonne et se caractérise par une forte présence de sujets de petits diamètres qui vont assurer la relève. Les conditions pédoclimatiques semblent assurer une bonne régénération de l'espèce. Ces résultats corroborent ceux de Adjonou et al. (2009); de Ouédraogo (2006) où les peuplements relativement stables de $A$. leiocarpa sont caractérisés par un nombre plus important de sujets appartenant aux classes [5$10[$ et [10-15[.

La structure instable de P. erinaceus, $L$. barteri, et $V$. paradoxa serait due à la combinaison des facteurs anthropiques et des conditions stationnelles. Les feux de brousse, le pâturage et les prélèvements à usage de bois de services sont des pratiques courantes dans les forêts claires de Koulbi. Aussi, dans ces formations où les cimes sont plus ou moins jointives, la compétition pour la lumière pourrait défavoriser certaines espèces comme $V$. paradaxa qui exploiterait mieux les savanes et les agrosystèmes.

\section{Régénération des principales espèces}

Les forêts claires offrent encore de bonnes conditions à la régénération des espèces ligneuses. L'espèce dominante $A$. leiocarpa régénère bien et la succession entre les classes de hauteurs présente un bon avenir. Ouédraogo et al. (2009) ont montré également que A. leiocarpa avait une bonne distribution dans les classes de hauteurs en dépit de la forte mortalité constatée entre le passage de la $1^{\text {ère }}$ classe ] 0-0,5 m ] à la classe suivante ] 0,5-1 m ]. $V$. paradoxa a également une bonne densité de régénération dans les forêts claires. C'est une espèce qui tente de s'adapter aux conditions des forêts claires, étant donné 
que la bonne partie de la semence est apportée par les frugivores. En effet, il est connu que le site de prédilection de la régénération reste la jachère (Kaboré et al., 2012). Les deux autres espèces ont une régénération faible. En outre, la succession entre les classes de hauteurs n'est pas assurée. Il est reconnu que $P$. erinaceus connaît des problèmes de régénération dans toutes les zones climatiques du Burkina Faso (Ouédraogo, 2006). Les changements climatiques, les feux de brousse et l'herbivorie seraient les facteurs responsables. Considérant toutes les espèces régénérant dans les forêts claires, la contribution des espèces caractéristiques $(37,43 \%)$ est faible. Ce qui voudrait dire que d'autres espèces peuvent caractériser ces sites dans le futur, si les conditions écologiques permettent leur développement.

\section{Menaces sur la survie des forêts claires}

Dans la forêt classée de Koulbi, l'agriculture constitue une menace pour la survie des forêts claires. Elles sont constamment défrichées pour la production agricole notamment celle de l'igname. Les stations de forêts claires semblent très propices à la production agricole. Du fait du tapi herbacée peu dense, la puissance des feux de brousse est atténuée, ce qui permet un dépôt important de litière qui se décompose.

A. leiocarpa, espèce dominante dans les forêts claires de Koulbi, connaît une mortalité de 9,27\% dont les causes restent encore peu élucidées. Tous les sujets morts étaient encore sur pied et nous avons noté aussi des individus mourant. Cela suggère que le phénomène est récent. Dans des conditions climatiques similaires à celles de la forêt classée de Koulbi, Adjonou et al. (2009) attribuait cette mortalité de A. leiocarpa à la réduction de la pluviométrie dans les forêts claires du Nord du Togo. L'espèce $A$. leiocarpa est caractérisée par une amplitude écologique exceptionnelle qui lui permet de survivre dans des milieux variables. $\mathrm{Au}$ Burkina Faso, elle est présente dans les quatre secteurs phytogéographiques du pays
(Ouédraogo et al., 2013). Bationo (1990) situe l'espèce entre les isohyètes $600 \mathrm{~mm}$ et 1100 $\mathrm{mm}$ au Burkina Faso. De ce postulat, il serait peu probable que les changements climatiques encours dans la zone d'étude soient directement responsables de cette mortalité. Cependant, l'augmentation de la température et la variation de l'humidité dans le milieu auraient créé un environnement favorable à la prolifération d'agents pathogènes, qui seraient responsables de la mortalité (Yáñez-López et al., 2012).

Par ailleurs, étant donné que $A$. leiocarpa a une faible capacité de reproduction séminale (Sacandé et Sanogo, 2007), il est donc probable que la multiplication végétative soit plus importante; ce qui lui confère un caractère grégaire (Thombiano et al., 2006). Dans de tels cas, la population présente une très faible diversité intra-spécifique car les individus sont issus d'un même pied mère. Une telle situation rend l'espèce vulnérable et favoriserait la prolifération d'agents pathogènes qui entraînent la dégénérescence de la population de A. leiocarpa des forêts claires.

\section{Conclusion}

L'analyse de la diversité floristique et de la dynamique des principales espèces fait ressortir que les îlots forêts claires de Koulbi ont une diversité floristique faible. Elles sont dominées par A. leiocarpa auquel s'associent $P$. erinaceus, L. barteri, $V$. paradoxa et $A$. digitata pour former la liste des espèces écologiquement importantes des forêts claires de Koulbi. Ces espèces écologiquement importantes influencent fortement (plus de $60 \%$ ) la densité et la surface terrière des arbres de ces formations. Par contre, elles ne contribuent qu'à environ $30 \%$ dans la régénération du peuplement.

L'analyse structurale de A. leiocarpa montre une bonne dynamique des sujets adultes et des sujets juvéniles. Cependant, l'espèce est confrontée à une mortalité $(9,27 \%)$ localisée qui serait causée par un agent pathogène. A cette menace, s'ajoute la 
pression anthropique encore forte sur ces formations qui existent sous forme d'îlots.

A la lumière de ces résultats, une étude plus approfondie s'avère nécessaire afin d'élucider les causes réelles de la mortalité de A. leiocarpa. Aussi, Il serait utile également de mettre en exergue l'importance de ces îlots dans la conservation de la faune de la forêt classée de Koulbi.

\section{REMERCIEMENTS}

Le projet Understanding and combating desertification to mitigate its impact on ecosystem-UNDESERT de l'Union Europenne, la direction provinciale de l'Environnement et des Ressources Halieutiques du Noubiel.

\section{REFERENCES}

Adjonou K, Bellefontaine R, Kokou K. 2009. Les forêts claires du Parc national OtiKéran au Nord-Togo: structure, dynamique et impacts des modifications climatiques récentes. Sécheresse, 20(1): 110 .

Adomou AC, MAMA A, MISSIKPODE R, SINSIN B. 2009. Cartographie et caractérisation floristique de la forêt marécageuse de lokoli (Bénin). Int. J. Biol. Chem. Sci., 3(3): 492-503.

Arbonnier M. 2009. Arbres, Arbustes et Lianes des Zones Sèches d'Afrique de l'Ouest ( $3^{\text {ème }}$ édn). Quæ Muséum Naturel d'Histoire Naturelle : Versailles.

Assogbadjo AE, Glele Kakaï RL, Sinsin B, Pelz D. 2009. Structure of Anogeissus leiocarpa Guill., Perr. Natural stands in relation to anthropogenic pressure within Wari-Maro Forest Reserve in Benin. Afr. J. Ecol., 48: 644-653.

Balma D, Bognounou O, Ouédraogo A, Tankoano MJ, Zigani G, Zigani M. 2004. La diversité biologique agricole au Burkina Faso. Archives de documents de la FAO p.21.

Bationo E, 1990. Etude de la distribution de Anogeissus leiocarpus (DC.) Guill. et Perr. et de Khaya senegalensis (Desr.) A.
Juss. Au Burkina Faso. Mémoire IDR, Université de Ouagadougou, p. 97.

Bellefontaine R, Gaston A, Petrucci Y. 2000. Management of Natural Forest of Dry Tropical Zones. Conservation Guide $\mathrm{n}^{\circ}$ 32. Food and Agri-cultural Organization (FAO) Rome.

Courtin F, Fournet F, Solano P. 2010. La crise ivoirienne et les migrants Burkinabé. L'effet boomerang d'une migration internationale, De Boeck Université/ Afrique Contemporaine, 4(236): 11-27.

Dossou ME, Lougbégnon OT, Houessou GL, Teka SO, Tente AHB. 2012. Caractérisation phytoécologique et structurale des groupements végétaux de la forêt marécageuse d'Agonvè et de ses milieux connexes au Sud-Bénin. Journal of Applied Biosciences, 53: 3821-3830.

Fontès J, Guinko S. 1995. Carte de la végétation et de l'occupation du sol du Burkina Faso. Notice explicative, p. 57.

Glélè Kakaï R, Akpona TJD, Assogbadjo AE, Gaoué OG, Chakeredza S,Gnanglè PC, Mensah GA, Sinsin B. 2011. Ecological adaptation of the shea butter tree (Vitellaria paradoxa C.F. Gaertn.) along climatic gradient in Benin, West Africa. Afr. J. Ecol., 49: 440-449.

Gouwakinnou GN, Kindomihou V, Assogbodjo A E, Sinsin B. 2009. Population structure and abundance of Sclerocarya birrea (A. Rich) Hochst subsp. birrea in two contrasting land-use systems in Benin. International Journal of Biodiversity and Conservation, 1(6): 194-201.

INSD. 2009. Annuaire statistique 2008. Ouagadougou, Burkina Faso, Institut national de la statistique et de la démographie, INSD, p. 413.

Kaboré SA, Bastide B, Traoré S, Boussim JI. 2012. Dynamique du karité, Vitellaria paradoxa, dans les systèmes agraires du Burkina Faso. Bois et Forêt des Tropiques, 313(3): 47-59. 
Mugnier J. 2008. Nouvelle flore illustrée du Sénégal et des régions voisines ( $1^{\text {ère }}$ édn) version électronique.

Nacoulma BMI. 2012. Dynamique et stratégies de conservation de la végétation et de la phytodiversité du complexe écologique du parc national du $\mathrm{W}$ du Burkina Faso. Thèse de doctorat, Université de Ouagadougou, Ouagadougou, p.151.

Ouédraogo A, Glèlè Kakaï R, Thiombiano A. 2013. Population structure of the widespread species, Anogeissus leiocarpa (DC.) Guill. \& Perr. across the climatic gradient in West Africa semi-arid area. South African Journal of Botany, 88: 286-295.

Ouédraogo A. 2006. Diversité et dynamique de la végétation ligneuse de la partie orientale du Burkina Faso. Thèse de doctorat de l'Université de Ouagadougou, Ouagadougou, p.195.

Ouédraogo O, Thombiano AK, Hahn-Hadjali K, Guinko S. 2009. Diversité et dynamique de la végétation ligneuse juvénile du Parc National d'Arly (Burkina Faso). Candollea, 64: 257-278.

Paré S. 2008. Land use dynamics, tree diversity and local perception of dry forest decline in Southern Burkina Faso, West Africa. Doctoral thesis, Swedish University of Agricultural Sciences, p.182.

Philip MS. 2002. Measuring Trees and Forests $\left(2^{\text {nd }}\right.$ edn). Cabi publishing: London.

Reitsma JM. 1988. Forest Vegetation in Gabon. Tropenbos Technical Series 1. Tropenbos Foundation: The Netherlands; 142.
Rondeux J. 1999. La Mesure des Peuplements Forestiers. Presses Agronomiques de Gembloux : Gembloux ; 1-521.

Ryniker KA, Bush JK, Van Auken OW. 2006. Structure of Quercus gambelii communities in the Lincoln National forest, New Mexico, USA. Forest Ecology and Management, 233: 69-77.

Sacandé M, Sanogo S. 2007. Anogeissus leiocarpus (DC.) Guill. \& Perr. Forest \& Landscape : Denmark (119): 2 p.

Sokpon N, Biaou SH, Ouinsavi C, Hunhyet O. 2006. Bases techniques pour une gestion durable des forêts claires du Nord-Bénin: rotation, diamètre minimal d'exploitabilité et régénération. Bois et Forêt des Tropiques, 287: 45-57.

Tankoano B. 2012. Analyse de la couverture ligneuse de la forêt classée de Koulbi à l'aide de la télédétection et des SIG. Mémoire, Université Polytechnique de Bobo-Dioulasso, p. 66.

Thombiano A, Schmidt M, Kreft H, Guinko S. 2006. Influence du gradient climatique sur la distribution des espèces de Combretaceae au Burkina Faso (Afrique de l'Ouest). Candollea, 61: 189-213.

Van Laar A, Akça A, 2007. Forest Mensuration. Springer: the Netherlands.

Yáñez-López R, Torres-Pacheco I, GuevaraGonzález RG, Hernández-Zul MI, Quijano-Carranza JA, Rico-García E. 2012. The effect of climate change on plant diseases. African Journal of Biotechnology, 11(10): 2417-2428.

Zarnock SJ, Dell TR. 1985. An evaluation of percentile and maximum likelihood estimators of Weibull parameters. Forest Sciences, 31: 260-268. 